\title{
Stock Values and Earnings Call Transcripts: a Dataset Suitable for Sentiment Analysis
}

\author{
Dexter Roozen \\ Tilburg University \\ drh.roozen@gmail.com
}

\author{
Francesco Lelli \\ Tilburg University \\ f.lelli@tilburguniversity.edu \\ https://francescolelli.info
}

\begin{abstract}
:
The dataset reports a collection of earnings call transcripts, the related stock prices, and the related sector index. It contains a total of 188 transcripts, 11970 stock prices, and 1196 sector index values. Furthermore, all of these data originated in the period 2016-2020 and are related to the NASDAQ stock market.

The data have been collected using Yahoo Finance and Thomson Reuters Eikon. Specifically, Yahoo Finance offered daily stock prices and traded volume. At the same time, Thomson Reuters Eikon has been used as source for the earnings call transcripts.

The dataset can be used as a benchmark for the evaluation of several NLP techniques as well as machine learning algorithms for understanding their potential for financial applications. Moreover, it is also possible to expand the dataset by extending the period in which the data originated following a similar procedure.
\end{abstract}

Keywords: dataset, stock, sentiment analysis, nlp, Nasdaq, stock prices

\section{Introduction:}

The dataset contains earning call transcripts and related stock process of 10 popular stocks of the Nasdaq index. These data have been collected for performing a set of "bag of words" analysis in order to evaluate possible correlation between them and stock process.

Several researches try to use yahoo finance Data in order to gather insight in stock prices and volume of trading. This is the case of Rao et all in [2] and authors in [3]. Some of the authors access directly to API. However, in recent times yahoo has made several changes and this practice is becoming more difficult.

Earning call transcript have been collected via Thomson Reuters Eikon. Several authors has use this approach. This is the case of the works presented in [4],[5],[6] and [7] However, the exact procedure for attaining the transcripts is not specified within these papers.

With this preprint we intend to formalize the procedure for collecting the data as well as release an initial version of a dataset that can be extended and reused in order to perform several different analysis.

The table below summarize the data: 


\begin{tabular}{|c|c|}
\hline Subject & Economics/Linguistics/Computer Science \\
\hline Specific subject area & $\begin{array}{l}\text { The specific subject area of this research is Sentiment Analysis. Sentiment } \\
\text { analysis is a natural language processing (NLP) technique to determine } \\
\text { the sentiment (positive or negative) behind data. } \\
\text { To elaborate, NLP is a field of research that investigates the ability of } \\
\text { computers to understand and manipulate natural languages, such as } \\
\text { English. } \\
\text { A crucial step of textual sentiment analysis is to pre-process the text } \\
\text { documents. This pre-processing phase consists of multiple 'pre- } \\
\text { processing techniques' of which the effects were studied. }\end{array}$ \\
\hline Type of data & $\begin{array}{l}\text { Table } \\
\text { Text }\end{array}$ \\
\hline How data were acquired & $\begin{array}{l}\text { The stock values and sector index were acquired through Yahoo Finance } \\
\text { (website). } \\
\text { The earnings call transcripts were acquired through Thomson Reuters } \\
\text { Eikon (software). }\end{array}$ \\
\hline Data format & Raw \\
\hline $\begin{array}{l}\text { Parameters for data } \\
\text { collection }\end{array}$ & $\begin{array}{l}\text { The related companies of the stock values and earnings call transcripts } \\
\text { were chosen based on the condition of being NASDAQ listed. } \\
\text { Furthermore, the date range for the stock values and earnings call } \\
\text { transcripts is } 2016-2020 \text {. }\end{array}$ \\
\hline $\begin{array}{l}\text { Description of data } \\
\text { collection }\end{array}$ & $\begin{array}{l}\text { The stock values were acquired by using Yahoo Finance. Yahoo Finance } \\
\text { provides news, information, commentary, and reports on the subject of } \\
\text { finance. This website lets users search for specific companies with its } \\
\text { search bar. When entering a company such as "Apple Inc.", the website } \\
\text { will direct the user to a summary of general financial information about } \\
\text { the company. Besides this 'Summary' tab, there are also other tabs } \\
\text { providing different sorts of information. Selecting the 'Historical Data' tab } \\
\text { shows the historical stock values of the searched company. Additionally, } \\
\text { it also lets users specify the date period and the frequency with which it } \\
\text { shows the stock values. Afterward, the presented stock values can be } \\
\text { downloaded as a Microsoft Excel Comma Separated Value (CSV) File. } \\
\text { Thomson Reuters Eikon helped acquire the earnings call transcripts. This } \\
\text { software provides users with many different sorts of financial }\end{array}$ \\
\hline
\end{tabular}




\begin{tabular}{|l|l|}
\hline & $\begin{array}{l}\text { information. Selecting the 'advanced event search' directs the user to } \\
\text { financial information about particular events. Specifying the event type } \\
\text { 'Earnings Conference Call' shows information about earnings calls of } \\
\text { many different companies. Selecting 'transcript' from the 'Content Type' } \\
\text { selector filters out earnings calls without transcripts. Lastly, date } \\
\text { preference and company should be specified to find the desired } \\
\text { information. With the save batch icon it is possible to download } 100 \\
\text { transcripts at a time as text documents. }\end{array}$ \\
\hline Data source location & $\begin{array}{l}\text { https://doi.org/10.34894/TJEODO } \\
\text { Data accessibility }\end{array}$ \\
$\begin{array}{l}\text { Repository name: DataverseNL } \\
\text { Data identification number: N.A } \\
\text { Direct URL to data: https://doi.org/10.34894/TJEODO }\end{array}$ \\
\begin{tabular}{l} 
Instructions for accessing these data: Data are open access \\
\hline
\end{tabular}
\end{tabular}

\section{Methods for data acquisition}

All of the stock values and the sector index were acquired by utilizing the Yahoo Finance search bar. Searching for a company such as Apple Inc. results in a summary of financial information about this company. However, the stock values and sector index within the dataset are presented in the "Historical Data" tab. Selecting this tab and specifying the time period January $1^{\text {st }}, 2016$ - October $1^{\text {st }}, 2020$ and selecting "Apply" will show the data presented in this dataset. Lastly, selecting "Download" provides a CSV file containing all of this data.

The earnings call transcripts were acquired through Thomson Reuters Eikon. Selecting the "advanced event search" option shows unfiltered financial information about many different sorts of events. Specifying the event type by selecting "Earnings Conference Call" will filter this information by only showing information about earnings calls. Additionally, selecting "Transcript" from the "Content Type" selector will show only earnings calls that can be provided together with a transcript of the earnings call. Lastly, specifying the company and time period will show a list with the earnings call transcripts contained in this dataset. For efficiency purposes, the save batch icon makes it possible to download this whole list of transcripts. 


\subsection{List of resources used for the collection of the data}

The following sources were used in retrieving the historical stock values of the NASDAQ listed companies and the sector index:

- NASDAQ. (2020). Apple Inc. (AAPL). [Historical stock values, 2016-2020]. Retrieved from https://finance.yahoo.com/quote/AAPL/history?p=AAPL

- NASDAQ. (2020). Advanced Micro Devices, Inc. (AMD). [Historical stock values, 2016-2020]. Retrieved from https://finance.yahoo.com/quote/AMD/history?p=AMD

- NASDAQ. (2020). Amazon.com, Inc. (AMZN). [Historical stock values, 2016-2020]. Retrieved from https://finance.yahoo.com/quote/AMZN/history?p=AMZN

- NASDAQ. (2020). ASML Holding N.V. (ASML). [Historical stock values, 2016-2020]. Retrieved from https://finance.yahoo.com/quote/ASML/history?p=ASML

- NASDAQ. (2020). Cisco Systems, Inc. (CSCO). [Historical stock values, 2016-2020]. Retrieved from https://finance.yahoo.com/quote/CSCO/history?p=CSCO

- NASDAQ. (2020). Alphabet Inc. (GOOGL). [Historical stock values, 2016-2020]. Retrieved from https://finance.yahoo.com/quote/GOOGL/history?p=GOOGL

- NASDAQ. (2020). Intel Corporation (INTC). [Historical stock values, 2016-2020]. Retrieved from https://finance.yahoo.com/quote/INTC/history?p=INTC

- NASDAQ. (2020). Microsoft Corporation (MSFT). [Historical stock values, 2016-2020]. Retrieved from https://finance.yahoo.com/quote/MSFT/history?p=MSFT

- NASDAQ. (2020). Micron Technology, Inc. (MU). [Historical stock values, 2016-2020]. Retrieved from https://finance.yahoo.com/quote/MU/history?p=MU

- NASDAQ. (2020). NVIDIA Corporation (NVDA). [Historical stock values, 2016-2020]. Retrieved from https://finance.yahoo.com/quote/NVDA/history?p=NVDA

- NASDAQ. (2020). NASDAQ Composite (^IXIC). [Historical stock values, 2016-2020]. Retrieved from https://finance.yahoo.com/quote/\%5EIXIC/history?p=\%5EIXIC

The following database provided all of the earnings call transcripts from the selected NASDAQ companies:

- Thomson Reuters Eikon. (2020). [Earnings call transcripts, 2016-2020]. Available at: Thomson Reuters (Accessed: November 19 2020). 


\section{Results: Data Description}

The folder named "Stock Values and Sector Index" in the dataset contains all of the CSV files that were acquired through the before mentioned method. These files consist of individual tables for each NASDAQ Company and the NASDAQ sector index. The folder is structured as portrayed in table 1.

\begin{tabular}{|l|c|l|l|}
\hline \multicolumn{1}{|c|}{ Company } & $\begin{array}{c}\text { File } \\
\text { Type }\end{array}$ & \multicolumn{1}{c|}{ Size } & Source \\
\hline Apple Inc. (AAPL) & CSV & 1197 rows $\times 1$ column & NASDAQ (2020) \\
\hline Advanced Micro Devices, Inc. (AMD) & CSV & 1197 rows $\times 1$ column & NASDAQ (2020) \\
\hline Amazon.com, Inc. (AMZN) & CSV & 1197 rows $\times 1$ column & NASDAQ (2020) \\
\hline ASML Holding N.V. (ASML) & CSV & 1197 rows $\times 1$ column & NASDAQ (2020) \\
\hline Cisco Systems, Inc. (CSCO) & CSV & 1197 rows $\times 1$ column & NASDAQ (2020) \\
\hline Alphabet Inc. (GOOGL) & CSV & 1197 rows $\times 1$ column & NASDAQ (2020) \\
\hline Intel Corporation (INTC) & CSV & 1197 rows $\times 1$ column & NASDAQ (2020) \\
\hline Microsoft Corporation (MSFT) & CSV & 1197 rows $\times 1$ column & NASDAQ (2020) \\
\hline Micron Technology, Inc. (MU) & CSV & 1197 rows $\times 1$ column & NASDAQ (2020) \\
\hline NVIDIA Corporation (NVDA) & CSV & 1197 rows $\times 1$ column & NASDAQ (2020) \\
\hline NASDAQ Composite (^IXIC) & CSV & 1196 rows $\times 1$ column & NASDAQ (2020) \\
\hline
\end{tabular}

Table 1: Folder structure stock values and sector index

Accessing these files can be done through Microsoft Excel. A snippet of what these files look like when opened in Excel is portrayed in figure 1. 


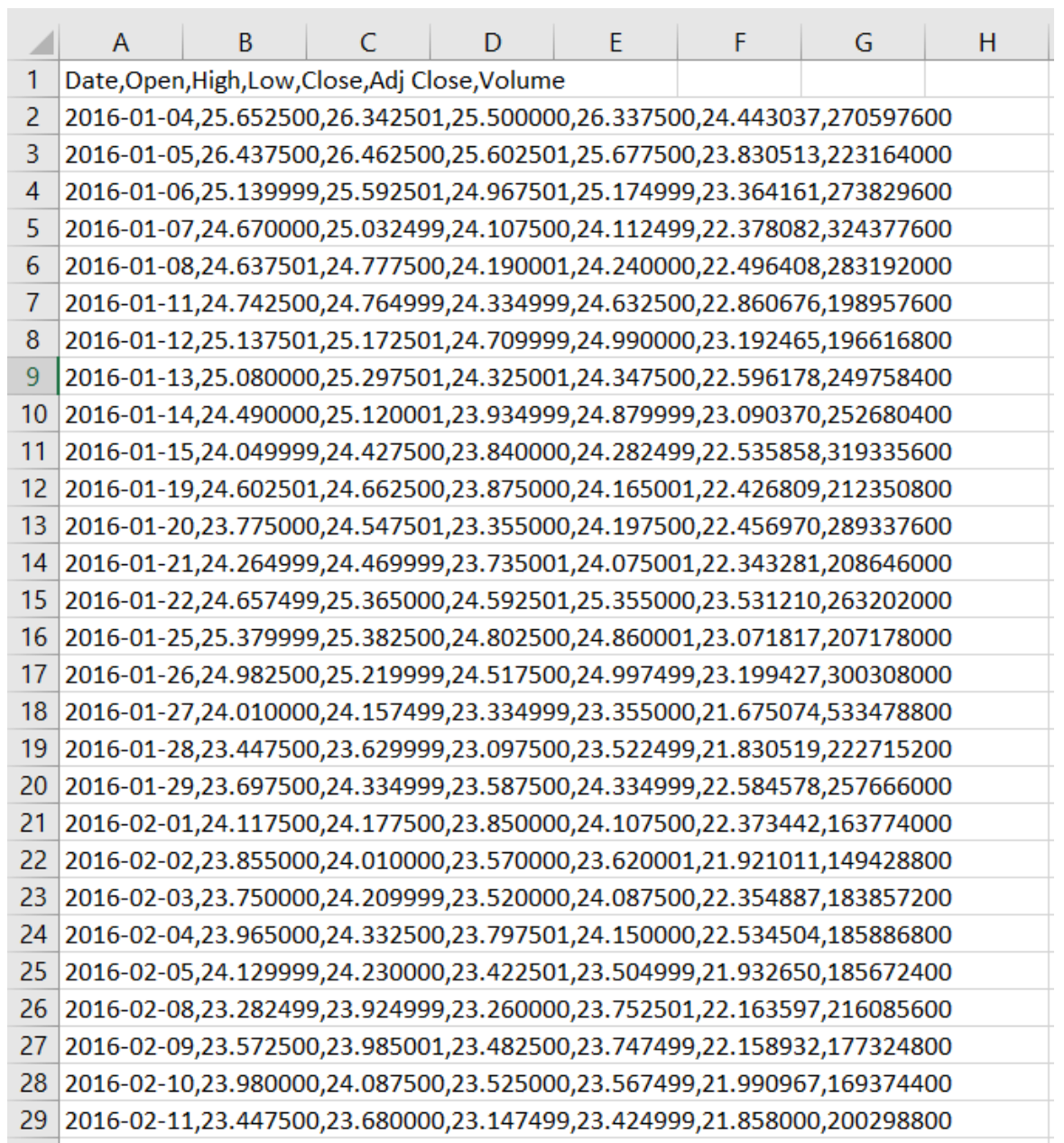

Figure 1: Stock values in Excel

Furthermore, the folder named "Transcripts" in the dataset contains multiple folders named after each company of which the earnings call transcripts were acquired. The structure of these folders is portrayed in table 2 .

\begin{tabular}{|l|l|l|}
\hline \multicolumn{1}{|c|}{ Company Folder } & \multicolumn{1}{c|}{ Contents } & \multicolumn{1}{c|}{ Source } \\
\hline Apple Inc. (AAPL) & 19 Text Documents & Thomson Reuters Eikon (2020) \\
\hline Advanced Micro Devices, Inc. (AMD) & 19 Text Documents & Thomson Reuters Eikon (2020) \\
\hline Amazon.com, Inc. (AMZN) & 19 Text Documents & Thomson Reuters Eikon (2020) \\
\hline ASML Holding N.V. (ASML) & 19 Text Documents & Thomson Reuters Eikon (2020) \\
\hline Cisco Systems, Inc. (CSCO) & 19 Text Documents & Thomson Reuters Eikon (2020) \\
\hline Alphabet Inc. (GOOGL) & 19 Text Documents & Thomson Reuters Eikon (2020) \\
\hline Intel Corporation (INTC) & 19 Text Documents & Thomson Reuters Eikon (2020) \\
\hline Microsoft Corporation (MSFT) & 19 Text Documents & Thomson Reuters Eikon (2020) \\
\hline Micron Technology, Inc. (MU) & 17 Text Documents & Thomson Reuters Eikon (2020) \\
\hline NVIDIA Corporation (NVDA) & 19 Text Documents & Thomson Reuters Eikon (2020) \\
\hline
\end{tabular}

Table 2: Folder structure transcripts 
The earnings call transcripts are text documents that are all structured in the same manner. Date, time, participants, and the words that are spoken during the earnings call are all registered within these documents. Accessing these data can be done through standard software such as Notepad on Windows devices. A snippet of what these documents look like in Notepad is portrayed in figure 2.

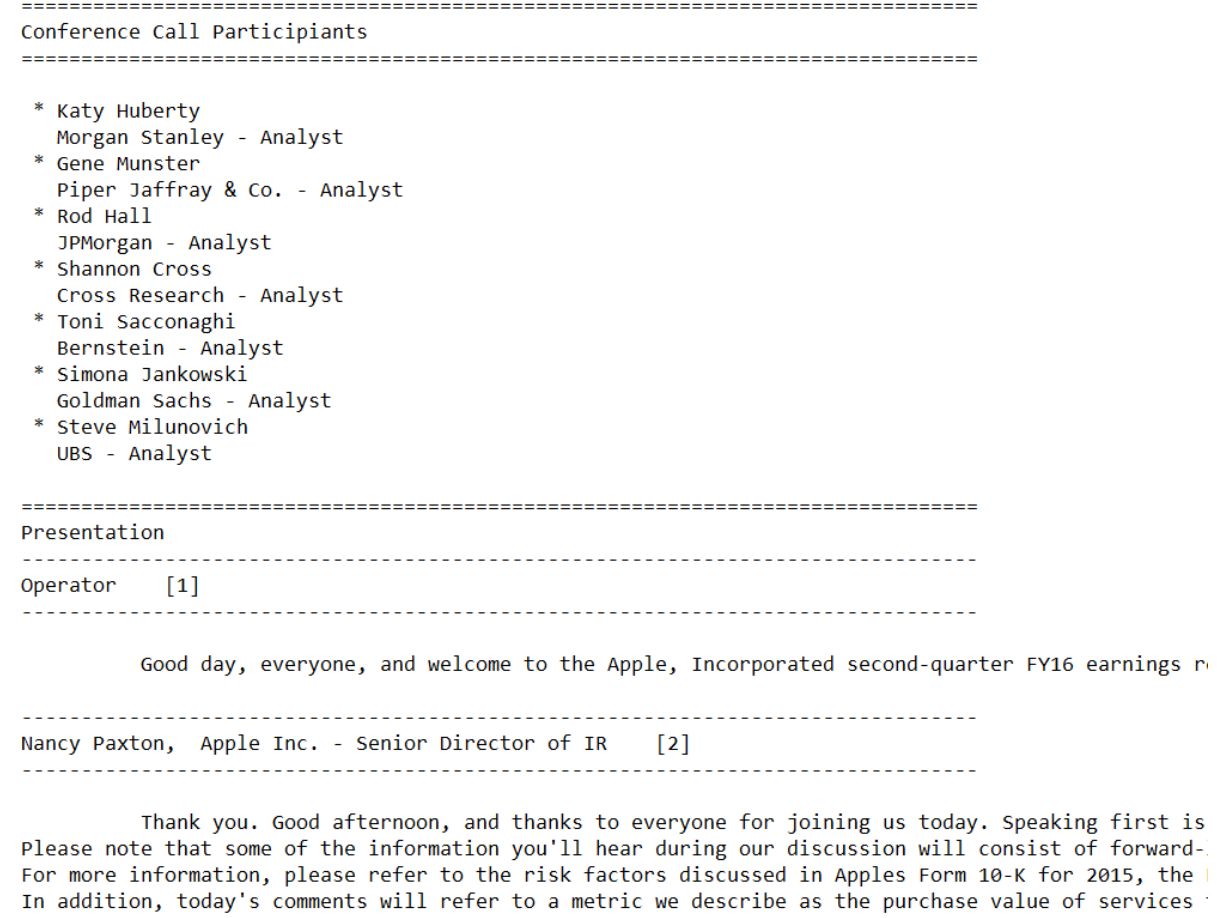

Figure 2: Snippet earnings call transcript

\subsection{Determining positive and negative transcripts}

Formulas are used to determine whether a transcript is positive or negative. Firstly, the stock ratio formula, which has the following form:

stock ratio = stock value one day after earnings call / stock value $n$ days before earnings call

The stock ratio shows the percentage increase or decrease of the stock value. However, a percentage increase in stock value does not immediately imply that the earnings call is positive as there are other variables to consider. To factor in an additional variable called investor mood, a second formula is defined:

sector ratio = sector value one day after earnings call / sector value $n$ days before earnings call

Sector refers to the NASDAQ composite. The sector ratio is taken into account to consider the mood of the sector index. If the increase in stock ratio turns out to be higher than the increase of the sector ratio, the earnings call can be determined positive. If not, the transcript is deemed negative. 


\section{Discussion}

The collected dataset provides the following value:

- These data can prove useful as they may help to further uncover dynamics related to correlational relationships between stock values and earnings call transcripts.

- Furthermore, the data can easily be expanded by i.e. extending the date range. Additionally, the data is easy to use and readable by multiple programming languages.

- Both practitioners at companies as well as scholars can benefit from the use of these data. Every company and scholar uses homemade datasets with consequential discrepancies. The adoption of a shared dataset for benchmarking analysis will promote a homogeneous evaluation of the results.

- The data was used primarily for the application of a limited amount of NLP techniques and machine learning algorithms. Consequently, this dataset offers the possibility to explore different approaches.

\section{Conclusions}

In this preprint we presented a dataset that has been designed for performing sentiment analysis in the stock market. Information regarding daily price and volume has been collected using yahoo finance. At the same, Thomson Reuters has been used for collecting earning transcripts. Details about the procedure has been described and presented in the previous sections. The dataset contains 11970 stock prices, and 1196 sector index values. Furthermore, all of these data originated in the period 2016-2020 and are related to the NASDAQ stock market.

The dataset can be used for developing and benchmarking NLP techniques and machine learning algorithms.

\section{Competing Interests}

The authors declare that they have no known competing financial interests or personal relationships which have or could be perceived to have influenced the work reported in this article.

\section{References}

[1] Roozen, D. R. H. (2021). Correlations between Earnings Call Transcripts and Stock Price: a Sentiment Analysis Approach. Master thesis at Tilburg University

[2] Rao, T., \& Srivastava, S. (2012). Analyzing stock market movements using twitter sentiment analysis. 
[3] Kordonis, J., Symeonidis, S., \& Arampatzis, A. (2016). Stock price forecasting via sentiment analysis on twitter. In Proceedings of the 20th Pan-Hellenic Conference on Informatics (pp. 1-6).

[4] Jha, V., Blaine, J., \& Montague, W. (2015). Finding Value in Earnings Transcripts Data with AlphaSense. Available at extractalpha.com

[5] Heinrichs, A., Park, J., \& Soltes, E. F. (2019). Who consumes firm disclosures? Evidence from earnings conference calls. The Accounting Review, 94(3), 205-231.

[6] Theil, C. K., Broscheit, S., \& Stuckenschmidt, H. (2019). PRoFET: Predicting the Risk of Firms from Event Transcripts. In IJCAI (pp. 5211-5217).

[7] Jenkins, P. (2020, April). Structured Paragraph Embeddings of Financial Earnings Calls. In Companion Proceedings of the Web Conference 2020 (pp. 264-268). 